\title{
Magnitude of poor Sleep Quality and Associated Factors among Health Sciences Students in Jimma University, Southwest Ethiopia, 2017
}

\author{
Mebrahtom Zeru ${ }^{1}$, Hiwot Berhanu ${ }^{1 *}$ and Andualem Mossie $^{2}$ \\ ${ }^{1}$ Department of Biomedical Sciences, Institute of Health, Ethiopia \\ ${ }^{2}$ Department of Biomedical Sciences, College of Medicine and Health Sciences, Ethiopia
}

*Corresponding author: Hiwot Berhanu, Department of Biomedical Sciences, Institute of Health, Jimma University, Ethiopia

\begin{tabular}{|c|}
\hline ARTICLE INFO \\
\hline Received: 幽 January 29, 2020 \\
\hline Published: 牶 February 07, 2020 \\
\hline
\end{tabular}

Citation: Mebrahtom Zeru, Hiwot Berhanu, Andualem Mossie. Magnitude of poor Sleep Quality and Associated Factors among Health Sciences Students in Jimma University, Southwest Ethiopia, 2017. Biomed J Sci \& Tech Res 25(3)-2020. BJSTR. MS.ID.004202.

Keywords: Poor Sleep Quality; University Students; Pittsburgh Sleep Quality Index

\begin{abstract}
Background: Sleep disorders have been notified as one of the main health problems in the society. Most university students suffer from poor sleep quality which is a direct cause for daytime malfunction. There is a scarcity of data about sleep quality and its associated factors among university students in Ethiopia. The main aim of this study is to determine the magnitude of poor sleep quality and its associated factors among health sciences students in Jimma University, Southwest Ethiopia.
\end{abstract}

Methods: A cross-sectional study was conducted from May27 to June 27; 2017.Multistage sampling technique was used to select 422 students. Data were collected using a semi-structured, pre-tested, self-administered questionnaire. EpiData version 3.1 and SPSS Version 20 for Windows were used for data entry and data analyses respectively. Frequencies, means, and proportions were used for descriptive data analysis. Multiple logistic regression was used to assess the association between poor sleep quality and independent variables. Statistical significance was taken at $\mathrm{P}<0.05$.

Results: A total of 404 students completed and returned the questionnaires making a response rate of $95.74 \%$. More than half of the respondents (54.2\%) were classified as having poor sleep quality. More males (66.7\%) were found to have poor sleep quality than females (33.3\%). However, the difference was not statistically significant ( $p$-value=0.62). Multiple logistic regression analysis revealed that as student's mean cumulative grade point average (CGPA) increases by one point, the probability to have poor sleep quality decreased by about $52 \%(\mathrm{OR}=0.48)$. Alcohol consumers were 2.5 times more likely to have poor sleep quality compared to their counterparts $[\mathrm{AOR}=2.5$, CI $(1.37-4.60) \mathrm{p}=$ 0.003]. Similarly, khat chewers were 2.5 times more likely to have poor sleep quality than non-chewers $[\mathrm{AOR}=2.5, \mathrm{CI}(1.26-4.93) \mathrm{p}=0.008]$. Those who use an electronic device for more than 2 hours a day were 3.9 times more likely to have poor sleep quality compared to those who use $<1$ hour a day $[\mathrm{AOR}=3.9$, CI $(1.33-11.64) \mathrm{p}=0.013]$.

Conclusions: Poor sleep quality was highly prevalent among Jimma University health science students. Alcohol consumption, khat chewing, academic performance, and electronic device use were the independent factors associated with poor sleep quality.

\section{Introduction}

Sleep is a naturally recurring state characterized by unconsciousness, relatively suspended sensory activity, and inactivity of nearly all voluntary muscles [1]. It is very important for growth and repair, memory consolidation and restorative processes to occur in the body [2,3]. Getting sufficient sleep is one of the crucial things that we can do to keep our body healthy [4]. It is required for optimal wellbeing and function of key cognitive functions related with academic performance in school $[5,6]$. However, the standard of living and environmental factors are causing sleep disturbance [7]. The National Sleep Foundation's recommend adolescents to 
get 8.5- 9.25 hours of sleep and adults to get 7-9 hours of sleep $[5,6]$. When students join university, they begin new living styles and most students modify their sleeping time and sleeping habits $[8,9]$. Which could be as a predisposing factor, for the increment of poor sleep quality among university students [10].

A study conducted to characterize sleep patterns and predictors of poor sleep quality in a large population of college student's shows over $60 \%$ were categorized as poor sleepers by the PSQI measurement [11]. The finding of different studies shows the prevalence of poor sleep quality among Asian medical student range from 19- 74.2\% [12-16]. Other studies conducted among Nigerian medical student shows the prevalence of sleep quality were $70 \%$ [17]. The prevalence of poor sleeps quality among colleges Ethiopian student by Lemma et al has been reported to be $55.8 \%$ [18]. Another study conducted in Debre Berhan University shows the prevalence of insomnia was $61.6 \%$ [19]. According to the findings of different studies female students get poor sleep quality relative to male students $[3,10,12,20,21]$. Another study showed that there was no statistically significant difference in sleep quality between males and females [22].

A study that evaluated the relationship between poor sleep quality and consumption of substances such as, alcohol, khat and cigarette showed a statistically significant association with poor sleep quality [20]. A study conducted in Jimma Ethiopia shows that chat chewers are more predisposed for poor sleep quality than non-chewers [23] Similarly, khat chewer students were classified as poor sleepers compared to those who did not chew khat. Khat chewing, especially at a higher quantity, also increased the risk of poor sleep quality, long sleep latency, poor sleep efficiency and sleep medicine use [20]. Students who reported the use of caffeinecontaining drinks per week were 1.48-times more likely to report long sleep latency compared to nonusers [20]. Smoking was positively correlated with poor sleep quality, Students who reported smoking cigarette had long sleep latency, poor sleep efficiency and sleep medicine use compared to nonsmokers [12,24].

Research conducted on the association of sleep quality and academic performance so far shows that poor quality sleep is associated with lower academic performance [25,26]. A study reported that there was statistically significant association between the use of electronic devices and poor sleep quality [8]. Prolonged Users of social networking, such as Face book had poorer sleep quality than Non-users [22]. Another study also found a positive relationship between the use of a mobile phone for playing games and chatting in bed with long sleep latency and severity of sleep disorders [24]. Limited number of studies was done to determine sleep quality and associated factors among medical students in Africa particularly in Ethiopia, there is scarcity of data that determine the magnitude and predictors of poor sleep quality, Therefore, we conduct this study so that it could fill the mentioned gaps by determining the prevalence of poor sleep quality and its associated factors among medical students.

\section{Methods and Materials}

The study was conducted at Jimma University, which is located in Jimma town $352 \mathrm{~km}$ Southwest of Addis Ababa, using a crosssectional study designs to determine the magnitude of sleep quality and associated factors among health science undergraduate regular students from May 27-June 27, 2017. The sample size was determined using the single population proportion formula, taking the following assumptions based on the previous study in the country that estimated prevalence of poor sleep quality among university students to be $52.7 \%$ [20], with confidence of $95 \%$, margin of error $5 \%$, and non-response rate of $10 \%$ were leading the final study sample of 422 student subjects. To reach at this sample the researchers deployed a Multi-stage sampling technique following which students from all courses of graduation were included in the study in order to maintain the representativeness of the sample. Students were stratified by field of study and batch (year of study).

After allocation of proportionate to size, the study subjects were selected by simple random sampling. Data were collected using a semi-structured, pre-tested, self-administered questionnaire developed by reviewing relevant literature and standard questionnaire used previously in similar studies (PSQI) and students' CGPAs were obtained from students' self-report and checked in the office of the registrar. Data were collected by BSc nursing who have training on data collection and previous experience of data collection under close supervision of two experienced supervisors. The questionnaire was initially prepared in English, translated to local languages Afaan Oromo and Amharic, and retranslated to English by another person who was blind to the original questionnaire for consistency check. Pretesting of the questionnaire was made on $5 \%$ of sample size among students of another university which is Walkite University (which was out of the main study area having (similar characteristics).

Data were edited, coded, and entered into Epi-Data version3.1 and exported to and analyzed by SPSS version 20. Frequencies, means, and proportions were used for the descriptive analysis of data. Tables and charts were used for data presentation. During an evaluation of association, P-value $<0.05$ was considered statistically significant for final result where $0.25 \mathrm{p}$-values was indicated to consider variables at screening stage for multi variable logistic regression. To make a research ethically safe \& acceptable, letter of permission was obtained from the Ethical clearance Committee of Jimma University. Similarly, at participant level the objectives of the study was explained to the participants and followed by written consent after the student agreed to participate with further confidentiality of the response by changing them anonymous.

\section{Results}

\section{Socio-Demographic Characteristics}

Out of 422 study subjects, 404 fully responded to the questionnaire making a response rate of $95.74 \%$. Majority of the 
participants were males (65.6\%), with a mean age of 22.15 years (SD \pm 2.1). More males (66.7\%) were found to have poor sleep quality than females (33.3\%). However, the difference was not statistically significant ( $p$-value $=0.62$ ). In relation to their ethnicity, Oromo $41.6 \%(n=168)$ account for highest proportion followed by Amhara $26.7 \%(n=108)$. Regarding the religion of the participants, Orthodox Christians account for highest proportion (44.3\%) followed by Protestant (28\%), Muslim (18.1\%), Catholic (5.7\%) and Traditions (3\%). Majority of the participants were single (96\%). Concerning field of study: medicine (53.2\%), pharmacy $(10.6 \%)$, nursing (18.1\%), anesthesia (4.2\%), environmental health (6.4\%), and medical laboratory (7.4\%).The proportion of poor sleep quality was highest among medicine students (56\%) followed by pharmacy students (53.7\%). In terms of study year, there were a high proportion of students who were studying in the fourth year (21.8\%) and second year (20.3\%). The proportion of participants categorized to have poor sleep quality was highest among sixthyear students (65.5\%) followed by the fifth year (56\%) and secondyear students $(56 \%)$. ( $\mathrm{SD}= \pm 0.48)$ (Table 1$)$.

Table 1: Socio-demographic characteristics of health science students in Jimma University, Ethiopia, 2017.

\begin{tabular}{|c|c|c|c|c|c|}
\hline Variable & Category & Frequency (\%) & Good sleep quality n (\%) & Poor sleep quality n (\%) & p-value \\
\hline Sex & $\begin{array}{l}\text { Male } \\
\text { Female }\end{array}$ & $\begin{array}{l}265(65.5) \\
139(34.4)\end{array}$ & $\begin{array}{c}119(64.3) \\
66(35.7)\end{array}$ & $\begin{array}{c}146(66.7) \\
73(33.3)\end{array}$ & 0.621 \\
\hline Marital status & $\begin{array}{c}\text { Single } \\
\text { Married } \\
\text { Divorced } \\
\text { Widowed }\end{array}$ & $\begin{array}{c}388(96) \\
14(3.5) \\
1(0.2) \\
1(0.2)\end{array}$ & $\begin{array}{c}180(97.3) \\
4(2.2) \\
1(0.5) \\
0(0)\end{array}$ & $\begin{array}{c}208(95) \\
10(4.6) \\
0(0) \\
1(0.5)\end{array}$ & 0.289 \\
\hline Department & $\begin{array}{c}\text { Medicine/Dentistry } \\
\text { Pharmacy } \\
\text { Nursing/Midwifery } \\
\text { Anaesthesia } \\
\text { Environmental Health } \\
\text { Medical Laboratory }\end{array}$ & $\begin{array}{c}218(54) \\
41(10.1) \\
73(18.1) \\
16(4) \\
26(6.4) \\
30(7.4)\end{array}$ & $\begin{array}{l}95(51.4) \\
19(10.3) \\
35(19) \\
8(4.3) \\
13(7) \\
15(8)\end{array}$ & $\begin{array}{c}122(55.7) \\
22(10) \\
38(17.4) \\
9(4) \\
13(6) \\
15(7)\end{array}$ & 0.97 \\
\hline Study year & $\begin{array}{l}\text { First Year } \\
\text { Second Year } \\
\text { Third Year } \\
\text { Fourth Year } \\
\text { Fifth Year } \\
\text { Sixth Year }\end{array}$ & $\begin{array}{l}78(19.3) \\
82(20.3) \\
76(18.8) \\
88(21.8) \\
48(11.9) \\
32(7.9)\end{array}$ & $\begin{array}{l}37(20) \\
36(19.5) \\
36(19.5) \\
43(23.2) \\
21(11.4) \\
12(6.5)\end{array}$ & $\begin{array}{c}41(18.7) \\
46(21) \\
40(18.3) \\
45(20.5) \\
27(12.3) \\
20(9)\end{array}$ & 0.904 \\
\hline
\end{tabular}

\section{Sleep Quality Parameters}

The average bedtime of the students, as reported on the PSQI, was at 11:40 PM (SD \pm 1:25 hour) and they wake up at 6:40 AM ( $S D \pm 00: 55$ hour). The mean total time to fall asleep was 24 minutes ( $S D \pm 14.78$ ). Participants' reported time in bed ranged from 3:30 to 13 hours with a mean time in the bed of 7:04 hours (SD \pm 1.50 ). Hours of reported actual sleep ranged from 3 to 12 hours with a mean total sleep time of 6.03 hours ( $S D \pm 1.46$ ). Majority of the participants $227(56.2 \%)$ had habitual sleep efficiency of $>85 \%$ with a calculated mean score of $85.6 \%$ (SD \pm 10.82 ). Nearly all $385(95.3 \%)$ of the participants had not taken medications to help them sleep in the past month. Two hundred seventy-eight (68.8\%) participants had no problems during the day due to their sleepiness (further measures of sleep quality are reported below as seen in Table 2. The global PSQI score of the participants ranged from 0 to 15. More than half of the respondents 54.2\% (219) were classified as having poor sleep quality (PSQI $>5$ ); at 95\%CI with a mean (SD) global score of 5.52(SD \pm 2.67$)$. 
Table 2: Pittsburgh sleep quality index (PSQI) subscales of health science students in Jimma University, Ethiopia, 2017.

\begin{tabular}{|c|c|c|}
\hline Variable & Category & Number (\%) \\
\hline \multirow{4}{*}{ Sleep duration (hours) } & $<5.0$ & $65(16.1)$ \\
\hline & $5.0-5.9$ & $107(26.5)$ \\
\hline & $6-6.9$ & $106(26.2)$ \\
\hline & $\geq 7$ & $126(31.2)$ \\
\hline \multirow{4}{*}{ Sleep latency (minutes) } & $\leq 15$ & $149(36.9)$ \\
\hline & $16-30$ & $202(50)$ \\
\hline & $31-60$ & $27(6.7)$ \\
\hline & $\geq 60$ & $26(6.4)$ \\
\hline \multirow{4}{*}{$\begin{array}{l}\text { Day dysfunction due to } \\
\text { sleepiness }\end{array}$} & Never & $278(68.8)$ \\
\hline & $<$ once a week & $91(22.5)$ \\
\hline & 1-2 times per week & $26(6.4)$ \\
\hline & $\geq 3$ times per week & $9(2.2)$ \\
\hline \multirow{4}{*}{ Sleep efficiency (\%) } & $\geq 85$ & $227(56.2)$ \\
\hline & $75-84$ & $125(30.9)$ \\
\hline & $65-74$ & $40(9.9)$ \\
\hline & $<65$ & $12(3)$ \\
\hline \multirow{4}{*}{$\begin{array}{l}\text { Sleep medicine during } \\
\text { past month }\end{array}$} & Never & $385(95.3)$ \\
\hline & $<$ once a week & $14(3.5)$ \\
\hline & 1-2 times per week & $4(1)$ \\
\hline & $\geq 3$ times per week & $1(0.2)$ \\
\hline
\end{tabular}

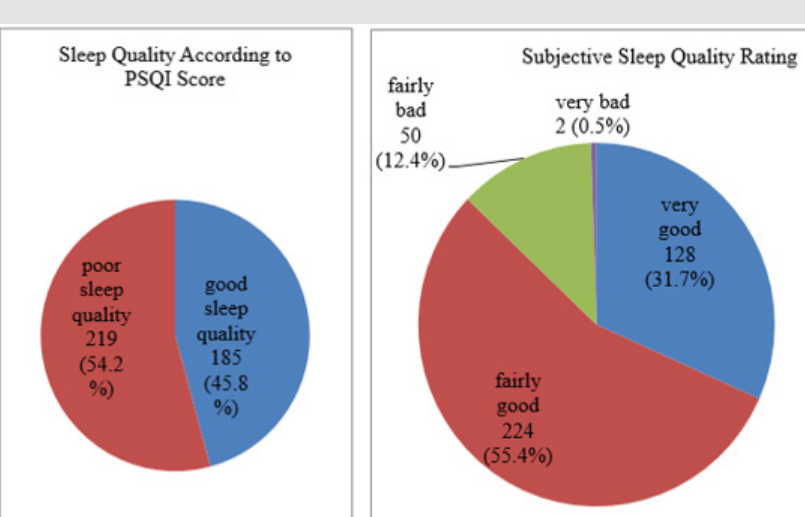

Figure 1: Sleep quality according to PSQI score and subjective sleep quality rating of health science students in Jimma University, Ethiopia, 2017.

Most of the participants (87\%) reported their sleep quality to be either very good or fairly good. When comparing subjective measures of self-rated sleep quality $(87 \%)$ reported either good or fairly good. Approximately $24.2 \%$ of the very good and $63 \%$ of the fairly good self-ratings for overall sleep quality had PSQI scores greater than five. Nearly all (90\%) of those who self-rated their sleep quality as fairly bad and all of those who self-rated their sleep quality as very bad were classified as having poor sleep quality using the PSQI scale (Figure 1). In the area of sleep disturbance, the study participants reported that, $11.6 \%(n=47)$ can't get asleep within 30 minutes, $29.5 \%(\mathrm{n}=119)$ wake up in the mid night, $22 \%$ ( $n=89)$ get up to use the toilet, $7.9 \%(n=32)$ can't take breaths comfortably, $12.4 \%$ (50) cough or sleep with harsh, $13.9 \%(n=56)$ feel too cold, $16.1 \%$ ( $n=65)$ experience bad dreams, and $52(12.9 \%)$ had pain/discomfort during sleep less than once a week (Table 3).

Table 3: Sleep disturbance symptoms of health science students in Jimma University, Ethiopia, 2017.

\begin{tabular}{|c|c|c|}
\hline Sleep disturbance symptom & Category & Number (\%) \\
\hline $\begin{array}{c}\text { Cannot get to sleep within } 30 \\
\text { minutes }\end{array}$ & $\begin{array}{c}\text { Never } \\
<\text { once a week } \\
1-2 \text { times per week } \\
\geq 3 \text { times per week }\end{array}$ & $\begin{array}{l}287(71) \\
47(11.6) \\
43(10.6) \\
27(6.7)\end{array}$ \\
\hline $\begin{array}{l}\text { Wake up in the middle of the } \\
\text { night or early morning }\end{array}$ & $\begin{array}{c}\text { Never } \\
<\text { once a week } \\
\text { 1-2 times per week } \\
\geq 3 \text { times per week }\end{array}$ & $\begin{array}{c}220(54.5) \\
119(29.5) \\
42(10.4) \\
23(5.7)\end{array}$ \\
\hline $\begin{array}{l}\text { Have to get up to use the } \\
\text { bathroom }\end{array}$ & $\begin{array}{c}\text { Never } \\
<\text { once a week } \\
1 \text {-2 times per week } \\
\geq 3 \text { times per week }\end{array}$ & $\begin{array}{c}262(64.9) \\
89(22) \\
39(9.7) \\
14(3.5)\end{array}$ \\
\hline Cannot breathe comfortably & $\begin{array}{c}\text { Never } \\
<\text { once a week } \\
1 \text {-2 times per week } \\
\geq 3 \text { times per week }\end{array}$ & $\begin{array}{c}360(89.1) \\
32(7.9) \\
9(2.2) \\
3(0.7)\end{array}$ \\
\hline Cough or snore loudly & $\begin{array}{c}\text { Never } \\
<\text { once a week } \\
1-2 \text { times per week } \\
\geq 3 \text { times per week }\end{array}$ & $\begin{array}{c}337(83.4) \\
50(12.4) \\
10(2.5) \\
7(1.7)\end{array}$ \\
\hline Feel too cold & $\begin{array}{c}\text { Never } \\
<\text { once a week } \\
1 \text {-2 times per week } \\
\geq 3 \text { times per week }\end{array}$ & $\begin{array}{c}328(81.2) \\
56(13.9) \\
11(2.7) \\
9(2.2)\end{array}$ \\
\hline Have bad dreams & $\begin{array}{c}\text { Never } \\
<\text { once a week } \\
1 \text {-2 times per week } \\
\geq 3 \text { times per week }\end{array}$ & $\begin{array}{c}306(75.7) \\
65(16.1) \\
19(4.7) \\
14(3.50\end{array}$ \\
\hline Have pain & $\begin{array}{c}\text { Never } \\
<\text { once a week } \\
1-2 \text { times per week } \\
\geq 3 \text { times per week }\end{array}$ & $\begin{array}{c}335(82.9) \\
52(12.9) \\
10(2.5) \\
7(1.7)\end{array}$ \\
\hline
\end{tabular}

\section{Substance Use and Electronic Device Use}

Substance in lifetime were $97.3 \%$ coffee/tea, 89.4\% Pepsi/ Coca-cola, $52 \%$ alcoholic beverages, $11.4 \%$ cigarette, and $28.5 \%$ 
khat; and the proportion of participants who reported substance use in the last month were 95\% coffee/tea, 70.5\% caffeinated cola products (Pepsi/Coca-cola), 30.9\% alcoholic beverages, 7.7\% cigarette, and $18.6 \%$ reported khat. Caffeinated beverages are the most frequently used substances; $67.3 \%$ respondents reported daily Coffee/ tea and $42.1 \%$ reported weekly coca-cola/pepsi drink. The proportion of participants categorized to have poor sleep quality was higher among substance users in the last month; coffee/ tea (54.4\%), coca-cola/pepsi (56.5\%), alcohol (70.4\%), cigarette $(80.6 \%)$, and khat $(76 \%)$ users were poor sleepers. Regarding electronic device, almost all 97.3\% respondents reported electronic device use and 264(65.3\%) of the participants use for more than 2 hours with mean (SD) duration of 247 minutes +/- 164.35 per day. Those who use an electronic device for more than 2 hours (63.2\%) had poorer sleep quality compared to those who use 1- 2 hours (41\%), and those who use less than 1 hour (34\%) a day (Table 4).

Table 4: Substance use and electronic device use of health science students in Jimma University, Ethiopia, 2017.

\begin{tabular}{|c|c|c|c|c|c|}
\hline Variable & Category & Number (\%) & Good sleep quality n (\%) & Poor sleep quality n (\%) & p-value \\
\hline Coffee/ tea drink in life time & $\begin{array}{l}\text { Yes } \\
\text { No }\end{array}$ & $\begin{array}{c}393(97.3) \\
11(2.7)\end{array}$ & $\begin{array}{c}180(97.3) \\
5(2.7)\end{array}$ & $\begin{array}{c}213(97.3) \\
6(2.7)\end{array}$ & 0.982 \\
\hline $\begin{array}{c}\text { Coffee/ tea drink in the last } 30 \\
\text { days }\end{array}$ & $\begin{array}{l}\text { Yes } \\
\text { No }\end{array}$ & $\begin{array}{c}384(95) \\
20(5)\end{array}$ & $\begin{array}{c}175(94.6) \\
5(2.7)\end{array}$ & $\begin{array}{c}209(95) \\
4(1.8)\end{array}$ & 0.552 \\
\hline $\begin{array}{l}\text { Frequency of Coffee/ tea drink in } \\
\text { the last } 30 \text { days }\end{array}$ & $\begin{array}{l}\text { Monthly or less } \\
\text { Weekly } \\
\text { Daily }\end{array}$ & $\begin{array}{c}33(8.2) \\
79(19.6) \\
272(67.3)\end{array}$ & $\begin{array}{c}17(9) \\
40(21.6) \\
118(64)\end{array}$ & $\begin{array}{c}16(7.3) \\
39(17.8) \\
154(70.3)\end{array}$ & 0.404 \\
\hline Coca-Cola/Pepsi drink in life time & $\begin{array}{l}\text { Yes } \\
\text { No }\end{array}$ & $\begin{array}{c}361(89.4) \\
43(10.6)\end{array}$ & $\begin{array}{c}168(91) \\
17(9)\end{array}$ & $\begin{array}{c}193(88) \\
26(12)\end{array}$ & 0.384 \\
\hline $\begin{array}{c}\text { Coca-Cola/Pepsi drink in last } 30 \\
\text { days }\end{array}$ & $\begin{array}{l}\text { Yes } \\
\text { No }\end{array}$ & $\begin{array}{l}285(70.5) \\
119(29.5)\end{array}$ & $\begin{array}{l}124(67) \\
45(24.3)\end{array}$ & $\begin{array}{c}161(73.5) \\
33(15)\end{array}$ & 0.026 \\
\hline $\begin{array}{l}\text { Frequency of Coca-Cola/Pepsi } \\
\text { drink in the last } 30 \text { days }\end{array}$ & $\begin{array}{c}\text { Monthly or less } \\
\text { Weekly } \\
\text { Daily or almost daily }\end{array}$ & $\begin{array}{c}94(23.3) \\
170(42.1) \\
23(5.7)\end{array}$ & $\begin{array}{c}46(24) \\
73(39.5) \\
6(3.2)\end{array}$ & $\begin{array}{c}48(22) \\
97(44.3) \\
17(7.8)\end{array}$ & 0.136 \\
\hline Alcohol drink in life time & $\begin{array}{l}\text { Yes } \\
\text { No }\end{array}$ & $\begin{array}{l}210(52) \\
194(48)\end{array}$ & $\begin{array}{c}75(40.5) \\
110(59.5)\end{array}$ & $\begin{array}{l}135(61.6) \\
84(38.4)\end{array}$ & $<0.001$ \\
\hline Alcohol drink in the last 30 days & $\begin{array}{l}\text { Yes } \\
\text { No }\end{array}$ & $\begin{array}{l}125(30.9) \\
279(69.1)\end{array}$ & $\begin{array}{c}37(20) \\
38(20.5)\end{array}$ & $\begin{array}{l}88(40.2) \\
47(21.5)\end{array}$ & 0.025 \\
\hline Alcohol type & $\begin{array}{c}\text { Beer } \\
\text { Wine } \\
\text { Local alcoholic bev- } \\
\text { erages }\end{array}$ & $\begin{array}{c}74(18.3) \\
16(4) \\
35(8.6)\end{array}$ & $\begin{array}{c}23(12.4) \\
3(1.6) \\
11(6)\end{array}$ & $\begin{array}{c}51(23.3) \\
13(6) \\
24(11)\end{array}$ & 0.705 \\
\hline $\begin{array}{c}\text { Frequency of Alcohol drink in the } \\
\text { last } 30 \text { days }\end{array}$ & $\begin{array}{c}\text { Monthly or less } \\
\text { Weekly } \\
\text { Daily or almost daily }\end{array}$ & $\begin{array}{l}58(14.4) \\
57(14.1) \\
10(2.5)\end{array}$ & $\begin{array}{c}23(12.4) \\
13(7) \\
1(0.5)\end{array}$ & $\begin{array}{c}35(16) \\
44(20) \\
9(4)\end{array}$ & 0.052 \\
\hline Cigarette smoking in life time & $\begin{array}{l}\text { Yes } \\
\text { No }\end{array}$ & $\begin{array}{c}46(11.4) \\
358(88.6)\end{array}$ & $\begin{array}{c}16(9) \\
169(91)\end{array}$ & $\begin{array}{c}30(13.7) \\
189(86.3)\end{array}$ & 0.111 \\
\hline $\begin{array}{c}\text { Cigarette smoking in the last } 30 \\
\text { days }\end{array}$ & $\begin{array}{l}\text { Yes } \\
\text { No }\end{array}$ & $\begin{array}{c}31(7.7) \\
373(92.3)\end{array}$ & $\begin{array}{c}6(3.2) \\
10(5.4)\end{array}$ & $\begin{array}{c}25(11.4) \\
8(3.7)\end{array}$ & 0.009 \\
\hline $\begin{array}{c}\text { Frequency of Cigarette smoking in } \\
\text { the last } 30 \text { days }\end{array}$ & $\begin{array}{c}\text { Monthly or less } \\
\text { Weekly } \\
\text { Daily or almost daily }\end{array}$ & $\begin{array}{c}4(1) \\
21(5.2) \\
7(1.7)\end{array}$ & $\begin{array}{l}1(0.5) \\
5(2.7) \\
1(0.5)\end{array}$ & $\begin{array}{l}3(1.4) \\
16(7.3) \\
6(2.7)\end{array}$ & 0.859 \\
\hline
\end{tabular}




\begin{tabular}{|c|c|c|c|c|c|}
\hline Khat chewing in life time & $\begin{array}{l}\text { Yes } \\
\text { No }\end{array}$ & $\begin{array}{l}123(30.4) \\
281(69.6)\end{array}$ & $\begin{array}{l}37(20) \\
148(80)\end{array}$ & $\begin{array}{l}86(39.3) \\
133(60.7)\end{array}$ & $<0.001$ \\
\hline Khat chewing in the last 30 days & $\begin{array}{l}\text { Yes } \\
\text { No }\end{array}$ & $\begin{array}{l}75(18.6) \\
329(81.4)\end{array}$ & $\begin{array}{l}18(9.7) \\
20(10.8)\end{array}$ & $\begin{array}{l}57(26) \\
30(13.7)\end{array}$ & 0.057 \\
\hline $\begin{array}{c}\text { Frequency of Khat chewing in the } \\
\text { last } 30 \text { days }\end{array}$ & $\begin{array}{c}\text { Monthly or less } \\
\text { Weekly } \\
\text { Daily or almost daily }\end{array}$ & $\begin{array}{l}22(5.4) \\
26(6.4) \\
27(6.7)\end{array}$ & $\begin{array}{l}9(5) \\
6(3.2) \\
3(1.6)\end{array}$ & $\begin{array}{l}13(6) \\
20(9) \\
24(11)\end{array}$ & 0.052 \\
\hline $\begin{array}{l}\text { Electronic device use in the last } \\
30 \text { days }\end{array}$ & $\begin{array}{l}\text { Yes } \\
\text { No }\end{array}$ & $\begin{array}{c}393(97.3) \\
11(2.7)\end{array}$ & $\begin{array}{c}179(96.8) \\
6(3.2)\end{array}$ & $\begin{array}{c}214(97.7) \\
5(2.3)\end{array}$ & 0.555 \\
\hline Duration of Electronic device use & $\begin{array}{l}<60 \text { minutes } \\
60-120 \text { minutes } \\
>120 \text { minutes }\end{array}$ & $\begin{array}{l}56(13.9) \\
73(18.1) \\
264(65.3)\end{array}$ & $\begin{array}{l}33(17.8) \\
53(28.6) \\
93(50.3)\end{array}$ & $\begin{array}{c}17(7.8) \\
37(17) \\
106(48.4)\end{array}$ & $<0.001$ \\
\hline
\end{tabular}

\section{Association Variables}

The odds of poor sleep quality according to participants' sociodemographic characteristics; substance use and an electronic device use were examined in the study. From a total of 15 variables initially considered to be probably associated with sleep quality; bivariate logistic regression analysis found 7 variables which fulfill the criteria $(\mathrm{p}<0.25)$. Age, monthly income, academic performance, alcohol consumption, cigarette smoking, khat chewing, and electronic device use were $p$-value $<0.25$ that enter to multiple logistic regression (Table 5). Multiple logistic regression analysis revealed that academic performance, Alcohol consumption, khat chewing, and electronic device use were significantly associated with sleep quality. Academic performance was negatively associated with poor sleep quality, as student's CGPA increases by one point, the probability to have poor sleep quality decreased by about $52 \%$ (AOR: 0.47 95\%CI: 0.26-0.86). Alcohol consumers were 2.5 times more likely to have poor sleep quality than non-consumers (AOR: 2.5 95\%CI: $1.37-4.60)$. Similarly, khat chewers were 2.5 times more likely to have poor sleep quality than their counterparts (AOR: 2.5 95\%CI: 1.26-4.92). Those who use electronic device $>2$ hours per day were 3.948 times more likely to be poor sleepers than those who use <1 hour per day (AOR: 3.94 95\%CI: 1.33-11.64) (Table 6).

Table 5: Bivariable and multivariable logistic regression model of factors independently associated with poor sleep quality among health science students in Jimma University, Ethiopia, 2017.

\begin{tabular}{|c|c|c|c|c|c|}
\hline Variable & Category & Total n (\%) & $\operatorname{COR}(95 \% \mathrm{CI})$ & AOR(95\% CI) & p-value \\
\hline \multicolumn{2}{|l|}{ CGPA } & 303 & $0 . .518(0.318-0.845)$ & $0.476(0.261-0.869)$ & 0.016 \\
\hline Coffee/ tea drink in life time & $\begin{array}{l}\text { Yes } \\
\text { No }\end{array}$ & $\begin{array}{c}393(97.3) \\
11(2.7)\end{array}$ & $\begin{array}{c}0.982(0.296-3.285) \\
1\end{array}$ & & \\
\hline Coca-Cola/Pepsi drink in life time & $\begin{array}{l}\text { Yes } \\
\text { No }\end{array}$ & $\begin{array}{c}361(89.4) \\
43(10.6)\end{array}$ & $\begin{array}{c}0.751(0 . .394-1.432) \\
1\end{array}$ & & \\
\hline Alcohol drink in life time & $\begin{array}{l}\text { Yes } \\
\text { No }\end{array}$ & $\begin{array}{l}210(52) \\
194(48)\end{array}$ & $\begin{array}{c}2.357(1.579-3.518) \\
1\end{array}$ & $\begin{array}{c}2.518(1.378-4.60) \\
1\end{array}$ & 0.003 \\
\hline Cigarette smoking in life time & $\begin{array}{l}\text { Yes } \\
\text { No }\end{array}$ & $\begin{array}{l}46(11.4) \\
358(88.6)\end{array}$ & $\begin{array}{c}1.677(0.883-3.184) \\
1\end{array}$ & $\begin{array}{c}0.496(0.185-1.325) \\
1\end{array}$ & 0.162 \\
\hline Khat chewing in life time & $\begin{array}{l}\text { Yes } \\
\text { No }\end{array}$ & $\begin{array}{l}123(30.4) \\
281(69.6)\end{array}$ & $\begin{array}{c}2.586(1.648-4.060) \\
1\end{array}$ & $\begin{array}{c}2.495(1.264-4.926) \\
1\end{array}$ & 0.008 \\
\hline $\begin{array}{l}\text { Electronic device use in the last } \\
\qquad 30 \text { days }\end{array}$ & $\begin{array}{l}\text { Yes } \\
\text { No }\end{array}$ & $\begin{array}{c}393(97.3) \\
11(2.7)\end{array}$ & $\begin{array}{c}1.435(0.431-4.779) \\
1\end{array}$ & & \\
\hline Duration of Electronic device use & $\begin{array}{l}<60 \text { minutes } \\
60-120 \text { minutes } \\
>120 \text { minutes }\end{array}$ & $\begin{array}{l}56(13.9 \\
73(18.1 \\
264(65.3\end{array}$ & $\begin{array}{c}1 \\
1.355(1.659-2.785) \\
3.340(1.764-6.324)\end{array}$ & $\begin{array}{c}1 \\
1.016(0.311-3.317) \\
3.95(1.339-11.644)\end{array}$ & $\begin{array}{l}0.979 \\
\mathbf{0 . 0 1 3}\end{array}$ \\
\hline
\end{tabular}


Table 6: Final model of actors associated with poor sleep quality of health science students in Jimma University, Ethiopia, 2017.

\begin{tabular}{|c|c|c|c|c|}
\hline Variable & Category & Number (\%) & AOR(95\% CI) & p-value \\
\hline \multicolumn{2}{|l|}{ CGPA } & 303 & $0.476(0.261-0.0869)$ & 0.016 \\
\hline Alcohol drink in life time & $\begin{array}{l}\text { Yes } \\
\text { No }\end{array}$ & $\begin{array}{l}210(52) \\
194(48)\end{array}$ & $\begin{array}{c}2.518(1.378-4.600) \\
1\end{array}$ & 0.003 \\
\hline Khat chewing in life time & $\begin{array}{l}\text { Yes } \\
\text { No }\end{array}$ & $\begin{array}{l}123(30.4) \\
281(69.6)\end{array}$ & $\begin{array}{c}2.495(1.264-4.926) \\
1\end{array}$ & 0.008 \\
\hline Duration of Electronic device use & $\begin{array}{l}<60 \text { minutes } \\
60-120 \text { minutes } \\
>120 \text { minutes }\end{array}$ & $\begin{array}{l}56(13.9) \\
73(18.1) \\
264(65.3)\end{array}$ & $\begin{array}{c}1 \\
1.016(0.311-3.317) \\
3.948(1.339-11.644)\end{array}$ & $\begin{array}{l}0.979 \\
\mathbf{0 . 0 1 3}\end{array}$ \\
\hline
\end{tabular}

\section{Discussion}

In this study, approximately $54.2 \%$ of the students were classified as poor sleepers. Overall, this finding agrees with prior reports which show over half of the study participants among university students had poor sleep quality according to PSQI criteria. For instance, a survey conducted in the Universities of Ethiopia (Gondar and Haramaya) reported that $52.7 \%$ of students were poor sleepers [22]. Similarly, 51.8\% of students in Chile [15] and $58 \%$ of students in Hong Kong [8] had poor sleep quality. The result of this study is lower than studies conducted in Nigeria where poor sleep quality were 70\% [17] among Pakistan students which was 64.24\% [27] Saudi Arabia. Students 76\% [28]. The reported variations between different studies may be influenced by different socioeconomic demands, cultural habits and academic pressures among the different population groups. In this study alcohol is main the predictor of poor sleep quality among medical students the study agree by research conducted by Van Reen et al and with Kenney et al. which alcohol consumption was associated with later bedtimes and rise times $[29,30]$ this alterations result from acute alcohol use include shorter sleep onset, a decrease in amount of rapid eye movement sleep, more stage 4 sleep in the first half of the night, an increase in wake during sleep, and an increase in delta electroencephalography power.

Research indicates an association between irregular selfreported sleep patterns and increased alcohol consumption. Specifically, self-report of insomnia is associated with a twofold increase in self-reported alcohol abuse. [31] Whereas this study disagrees with the study done among large portion of college student by revealed that tension and stress accounted poor sleep quality, whereas alcohol and caffeine consumption, were not significant predictors of sleep quality [32]. Current khat chewers' participants had increased risk of poor sleep quality than non-chewers. This finding agrees with the study conducted in Jimma town and Yemen $[33,34]$ this could be the result of sympathomimetic effects of khat (Catha edulis) which has amphetamine like effect Which causes psycho-stimulation and euphorigenic effects by impairing the sleep center $[34,35]$. This study also investigated the use of an electronic device in relation to the quality of sleep among health science students. Most of the participants in this study reported the use of an electronic device.

In this study population, using an electronic device for $>2$ hours increased the risk poor sleep quality. Use of electronic devise for prolonged time showed relation with poor sleep quality, study conducted among southern Taiwan and Iran students demonstrated significant negative association between the degree of internet addiction and sleep quality $[36,37]$ The possible mechanism for the negative effect of electronics use on sleep is that the light produced by electronic devices may disturb circadian rhythms by inhibiting melatonin hormone, resulting in the inability to start sleep at the right time and the brightness of the light that is projected onto the retina from computers or mobile screens send signals to hypothalamus which is thought to be factors that trigger changes in sleep patterns [38].

\section{Limitation and Strength of the Study}

Even though we deployed a Multi-stage sampling technique we didn't use design effect. Second, use of a self-administered survey that relied on subjective measures of sleep quality and other covariates may have introduced some degree of error in reporting behavioral covariates, and the period of the semester when the survey was administered could have influenced the sleep quality. However, we believe that by the use of the anonymous questionnaire and validated instrument might overcome these issues.

\section{Conclusion}

This study provides strong evidence that poor sleep quality is vastly prevalent among Jimma university health science students. The findings in this study show that alcohol consumption, khat chewing, duration of electronic device use and academic performance are statistically significant predictors of poor sleep quality which have a big impact on student's wellbeing. As sleep good quality may be a marker for health status and quality of life, prospective studies are needed to be done.

\section{Conflict of Interests}

The authors have not declared any conflict of interests. 


\section{Acknowledgement}

We would like to express our appreciations to Jimma University for financial support that helped us to undertake this research. We also express our heartfelt gratitude to all study participants and data collectors who were willing to participate in this study.

\section{References}

1. Taylor DJ, Bramoweth AD (2010) Patterns and Consequences of Inadequate Sleep in College Students: Substance Use and Motor Vehicle Accidents. Journal of Adolescent Health 46(6): 610-612.

2. Curcio G, Ferrara M, De Gennaro L (2006) Sleep loss, learning capacity and academic performance. Sleep Medicine Reviews.

3. Araújo MFM de, Lima ACS, Araújo TM de, Veras VS, Zanetti ML, et al. (2014) Association of sociodemografic factors and sleep quality in brazilian university students. Texto e Contexto Enfermagem, 23(1): 176184.

4. Boysan M (2016) Developmental Implications of Sleep. A J Clin Neurosci Psychopathol 18(2): 44-52.

5. (2007) Prince R, Hospital A. Global perspectives on sleep and health issues. pp. 35-42.

6. Afandi O (2013) Sleep Quality Among University Students: Evaluating the Impact of Smoking, Social Media Use , and Energy Drink Consumption on Sleep Quality and Anxiety. Student Pulse 5(06): 1-3.

7. Kazim M, Abrar A (2011) Sleep patterns and academic performance in pa students of a medical college in p. KUST Med J 3(2): 60

8. Azad MC, Fraser K, Rumana N, Abdullah AF, Shahana N, et al. (2015) Sleep disturbances among medical students: a global perspective. J Clin Sleep Med 11(1): 69-74.

9. Afandi O, Hawi H, Mohammed L, Salim F, Hameed AK, Rizwana B (2012) Sleep quality among University students in the UAE. GULF Med J 1: 193 199.

10. (2004) Regional WHO. WHO technical meeting on sleep and health European Centre for Environment and Health: 22-24.

11. Suen LK, Tam WW, Hon K (2010) Association of sleep hygiene-related factors and sleep quality among university students in Hong Kong. Hong Kong Med J 16(3): 180-185.

12. Lund HG, Reider BD, Whiting AB, Prichard JR, et al. (2010) Sleep Patterns and Predictors of Disturbed Sleep in a Large Population of College Students. J Adolesc Heal [Internet] 46(2): 124-132.

13. Lu J, Fang GE, Shen SJ, Wang Y, Sun Q (2011) A Questionnaire survey on sleeping in class phenomenon among Chinese medical undergraduates. Med Teach 33(6): 508

14. Huen LL, Chan TW, Yu WM, Wing YK (2007) Do medical students in Hong Kong have enough sleep? Sleep Biol Rhythms 5(3): 226-230.

15. Zailinawati AH, Teng CL, Chung YC, Teow TL, Lee PN, et al. (2009) Daytime sleepiness and sleep quality among Malaysian medical students. Med J Malaysia 64(2): 108-110.

16. Ghoreishi A (2008) Sleep quality in Zanjan university medical students. Tehran Univ Med J 66(1): 66-71.

17. Aesha Farheen Siddiqui, Hasan Al Musa, Hasan Al Amri, Abdulkareem Al Qahtani, Mushabab Al Shahrani, et al. (2016) Sleep Patterns and Predictors of Poor Sleep Quality among Medical Students in King Khalid University, Saudi Arabia Malays J Med Sci 23(6): 94-102.

18. Ibrahim NK, Badawi FA, Mansouri YM, Ainousa AM, (2017) Sleep Quality among Medical Students at King Abdulaziz University: A Cross-sectional Study. J Community Med Health Educ 7: 561.

19. Seblewngel Lemma, Bizu Gelaye, Yemane Berhane, Alemayehu Worku, Michelle A Williams (2012) Sleep quality and its psychological correlates among university students in Ethiopia: a cross-sectional study Lemma et al. BMC Psychiatry 12: 237.

20. Haile YG, Alemu SM, Habtewold TD (2017) Insomnia and Its Temporal Association with Academic Performance among University Students : A Cross-Sectional Study 4: 1-7.

21. Lemma S, Sheila V, Yared A, Mahlet G, Yemane B, et al. (2012) The epidemiology of sleep quality, sleep patterns, consumption of caffeinated beverages, and khat use among Ethiopian college students. Sleep Disorders: 593510.

22. Doi Y, Minowa M (2003) Gender differences in excessive daytime sleepiness among Japanese workers. SocSci Med 56(4): 883-894.

23. Afandi O, Hawi H, Mohammed L, Salim F, Hameed AK, Rizwana B (2012) Sleep quality among University students in the UAE. Gulf Med J 1: 193199.

24. Tadeg J, Andualem M, Hiwot B Yigizie Y (2019) Poor sleep quality and its predictors among type 2 diabetes mellitus patients attending Jimma University Medical Center, Jimma, Ethiopia. BMC Res Notes 12: 488.

25. Fossum IN, Nordnes LT, Straume S, Bjorvatn B, Pallesen S (2015) The Association Between Use of Electronic Media in Bed Before Going to Sleep and Insomnia Symptoms, Daytime Sleepiness, Morningness, and Chronotype. Behav Sleep Med 12(5): 343-357.

26. Gilbert SP, Weaver CC (2010) Sleep quality and academic performance in university students: a wake-up call for college psychologists. J Coll Student Psychother: 295-306.

27. Perkinson Gloor N, Lemola S, Grob A (2013) Sleep duration positive attitude toward life, and academic achievement: The role of daytime tiredness, behavioral persistence, and school start times. J Adolesc 36(2): 1-8.

28. Maheshwari G, Shaukat F (2019) Impact of Poor Sleep Quality on the Academic Performance of Medical Students. Cureus 11(4): e4357.

29. Almojali, Almalki SA, Alothman AS, Masuadi EM, Alaqeel MK (2017) The prevalence and association of stress with sleep quality amongmedical student. Journal of Epidemiology and Global Health 7(3): 169-174.

30. Van Reen E, Roane BM, Barker DH, McGeary JE, Borsari B, et al. (2016) Current alcohol use is associated with sleep patterns in first-year college students. SLEEP 39(6): 1321-1326.

31. Brower KJ (2003) Insomnia, alcoholism and relapse. Sleep Med Rev $7(6): 523-539$.

32. Hannah G Brian D (2010) Sleep Patterns and Predictors of Disturbed Sleep in a Large Population of College Students. Journal of Adolescent Health 46(2): 124-132

33. Berhanu H, Mossie A, Tadesse S, Geleta D (2018) Prevalence and associated fac-tors of sleep quality among adults in Jimma Town, Southwest Ethiopia: a community-based cross-sectional study. Sleep Disord 4: 342328 .

34. AD Ali (2015) A pilot community-based study on association ofKhat (Catha edulis) chewing practice with psychosis in Yemen. American Journal of Health Research 3(2): 91-96.

35. Abebe M, Adane K (2015) Adverse health efects of khat: a review. Family Med Sci Res 4(1): 154.

36. Lin PH, Lee YC, Chen KL, Hsieh PL, Yang SY, et al. (2019) The Relationship Between Sleep Quality and Internet Addiction Among Female College Students. Front Neurosci 13: 599.

37. Purreza A, Mostafa E, Saeed R, Moslem J, (2016) Studying the Relationship between Quality of Sleep and Addiction to Internet among Students. Nova Journal of Medical and Biological Sciences 5(3): 1-7.

38. Cheung LM, Wong WS (2011) The effects of insomnia and internet addiction on depression in Hong Kong Chinese adolescents: an exploratory cross-sectional analysis. Journal of Sleep Research 20(2): 311-317. 


\section{ISSN: 2574-1241}

DOI: $10.26717 /$ BJSTR.2020.25.004202

Hiwot Berhanu. Biomed J Sci \& Tech Res

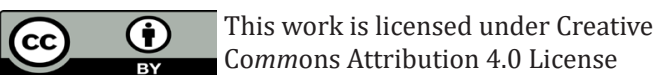

Submission Link: https://biomedres.us/submit-manuscript.php

$\begin{array}{ll}\text { BIOMEDICAL } & \text { Assets of Publishing with us } \\ \text { RESEARCHES } & \text { - Global archiving of articles } \\ \text { - Immediate, unrestricted online access } \\ \text { - Rigorous Peer Review Process }\end{array}$

